\title{
Radiological and functional lung sequelae of COVID-19
}

The identification of the novel coronavirus SARS-CoV-2 in December 2019, and the subsequent declaration of a global COVID-19 pandemic in March 2020 by the World Health Organization, has had far-reaching and unprecedented effects on global health and economics, and has dominated both clinical practice and research for pulmonologists. Many articles on COVID-19 and the immediate consequences of pneumonia caused by the disease have been published, but very few studies have investigated the long-term sequelae of COVID-19 pneumonia.

A systematic review and meta-analysis by So et al. ${ }^{[1]}$ investigated radiological and pulmonary function test abnormalities in patients who were evaluated after hospital discharge. The authors searched PubMed and EMBASE and identified 29 suitable studies (both prospective and retrospective). However, only 15 were evaluable: of these, 13 contained follow-up chest computed tomography data, and 10 studies contained follow-up pulmonary function data. Overall, 3066 patients were included in this report.

Age and sex, comorbidities, initial COVID-19 symptoms, residual COVID-19 symptoms after hospital discharge, follow-up timing, disease severity, chest computed tomography (CT) findings at follow-up and type of pulmonary function test abnormalities were all variables reported on. The mean age of the patients was 56 years, and $54.2 \%$ were male. Hypertension and diabetes were the two mostfrequently reported comorbidities. Radiological abnormalities were present on CT in $55.7 \%$ of patients after discharge, of which ground glass opacities (44.1\%) and parenchymal bands/fibrous bands (33.9\%) were the most frequent findings. Interestingly, this was despite the fact that only a small proportion of patients $(22.4 \%)$ had severe disease (defined as a requirement for high-flow oxygen, or invasive or noninvasive ventilation). Pulmonary function testing was abnormal in $44.3 \%$ of patients, and the most frequent abnormality was a decreased diffusion capacity of the lungs for carbon monoxide $\left(\mathrm{DL}_{\mathrm{CO}} \leq 70 \%\right)$ in $34.8 \%$ of patients). Restrictive and obstructive features were noted in $16.4 \%$ and $7.7 \%$ of cases, respectively.

One weakness of the study was the heterogeneity in methodology used to determine the end of COVID (symptoms v. polymerase chain reaction (PCR) negativity), and therefore uncertainty about whether imaging was performed during the resolution phase. Another weakness was differences in terminology used to describe CT abnormalities in the various studies. Lastly, comprehensive assessments of effort tolerance or of dyspnoea were not recorded in all studies.

Our understanding of the natural history and consequences of COVID pneumonia (and any persistent radiological or physiological changes that may result - part of so-called 'long COVID') is growing, and this study adds to this. We eagerly await further studies on what the functional impairment in survivors of severe COVID might be, and what additive effect post-COVID-19 lung disease might have on the already considerable burden of chronic lung disease in our country.

\section{Q Gurirab}

Department of Pulmonology, University of Cape Town and Groote Schuur Hospital, Cape Town, South Africa

\footnotetext{
1. So M, Kabata H, Fukunaga K, Takagi H, Kuno T. Radiological and functional lung sequelae of COVID-19: A systematic review and meta-analysis. BMC Pulm Med 2021;21(1):97. https://doi.org/10.1186/s12890-021-01463-0
} 